\title{
What Did it Cost?
}

\author{
ASHLEY BIGHAM \\ Ohio State University
}

Over drinks and hors d'oeuvres at the 107th Annual ACSA conference in Pittsburgh, an interesting conversation emerged among several young faculty members who teach at different public institutions. While reflecting on presentations of design projects each had seen earlier in the day, a common recurring question emerged: "What did it cost?" While each presentation had beautifully articulated the formal, aesthetic or material ambitions of its project, very few mentioned how much the projects had cost. Actually, none did.

For this Call to Action, I organized a discussion session which focused on workshopping a series of actual budgets with architects who run small, academic-based practices. Through an act of radical transparency, this positive working session aimed to produce tangible best practices and trade secrets on how, exactly, we manage the financial and logistical aspects of our work. Furthermore, the session encouraged file sharing of spreadsheet budgets, timesheets and other logistical documents as well as discussions on file organization, naming conventions, and office policies. As mundane as these tasks first appear, they are often the key to success in practices where frugality and efficiency are paramount to success.

Secrecy is often a tool for exclusion. If there is a need for a Call to Action in architecture today, it is to forgo the exclusive hierarchies of a previous generation, and empower young architectural practices with the tools and methods currently only learned through years of trial and error. With the current desire for more transparency and disclosure around professional issues of hiring and equal pay, this interactive panel applied the same openness and positive conversation to the internal workings of faculty-led firms. To combat unpaid labor, class exclusion, and closed-door agreements in the profession (and in academia) it is imperative that we quite literally "open the books" of our firms. The session will include a frank discussion of both the successes and mistakes we have each made in order to develop more successful practices moving forward.
In professional practice, public building budgets are often disclosed as part of the architect selection process and private residential projects may be associated with a typical price/ sq.ft. cost based on the type of construction. By contrast, in the world of academia-research exhibitions, speculative projects, and design competitions-it is often less clear how small practices manage project budgets, pay for student labor, afford materials, and secure grants. As faculty on the forefront of preparing our students for the profession of architecture (mentoring them against unpaid internships or advising on salary negotiation), we are not always the most knowledgable source for advice.

The following pages of this essay are dedicated to documents: a worksheet you can use to start your own working group on budgets, actual budgets for small-scale installations, exhibitions, and research projects. Some of these projects were completed, others remain speculative. 


\section{What Did It Cost?}

ACSA Fall Conference 2019

Working Session

1. Project: What was it?

a. What was the size, scope, and/or site of the project?

b. Was there a client? Who was the client?

c. How was the client related to the funding?

\section{Money: What did it cost?}

a. What was the original budget?

b. What role did your university play?

c. What role did your private practice play?

d. Did the project total stay within the original budget or were additional funds added?

e. How did the initial budget discussions come about and what would you have done differently about that conversation?

f. How do you manage out-of-pocket expenses? How were budget overruns covered?

3. Rules: What are the rules restricting how money is spent?

a. What are the legal entities involved in the project?

b. Can you/did you make a profit?

c. Can you/did you pay yourself for labor?

d. Can you/did you shift money from different projects?

\section{Resources: Who? How?}

a. What were your experiences with employees, etc?

b. What tools (fabrication equipment, etc.) did you utilize at your university or institution?

c. How did you manage liability insurance, payroll, etc.?

d. Did you receive donations of materials from corporations or other institutions?

\section{Conflicts: What are common project conflicts?}

a. What conflicts developed with the clients or sponsors of the project? With employees?

b. Do you encounter any ethical questions related to project finances?

\section{Outcomes: What are some of the "lessons learned" from this project?}

a. What would you do differently regarding the financial aspects of the project?

b. How will you approach the next project based on what you learned?

\section{Models: Who have you learned from?}

a. Where do you get the best advice on financial aspects of projects?

b. What practices do you know who are good at securing funding, utilizing resources, etc.?

c. Where else should we be looking for advice? 


\begin{tabular}{lrrrr}
$\begin{array}{l}\text { Spring Travel 2018: Faculty Budget } \\
\text { Housing }\end{array}$ Nights & \multicolumn{2}{c}{ City Total } & Cost per Night \\
& Kyiv & 6 & $\$ 870.00$ & $\$ 145$ \\
Kharkiv & 3 & $\$ 225.00$ & $\$ 75$ \\
Lviv & 5 & $\$ 500.00$ & $\$ 100$ \\
Yerevan & 6 & $\$ 960.00$ & $\$ 160$ \\
Tblisi & 6 & $\$ 660.00$ & $\$ 110$ \\
Housing Total & & & $\$ \mathbf{\$ 3}, \mathbf{2 1 5 . 0 0}$ \\
Total Days & $\mathbf{2 6}$ & &
\end{tabular}

\section{Transportation}

International Transportation

Ann Arbor>Detroit (roundtrip) $\$ 140.00$

Detroit>Kyiv (Round-trip to Europe) $\$ \$ 1,597.00$

Flight Tbilisi>Kyiv $\quad \$ 150.00$

Inter-city

$\begin{array}{rr}\text { Yerevan>Tbilisi } & \$ 35.00 \\ \text { Kyiv>Kharkiv } & \$ 50.00 \\ \text { Kharkiv>Lviv } & \$ 50.00\end{array}$

Intra-city

Transportation to Sevan $\$ 40.00$

Transportation to Khakveli $\quad \$ 40.00$

Transportation to Jvari Monastery $\quad \$ 40.00$

Transportation to Chernobyl $\$ \$ 100.00$

Transportation Total $\quad \$ \mathbf{\$ 2 , 2 4 2 . 0 0}$

Events

Entry Fees (museum, theater, etc.)

$\$ 40.00$

$\begin{array}{ll}\text { Events Total } & \$ \mathbf{\$ 4 0 . 0 0}\end{array}$

Misc.

Cell Phones (international access) $\quad \$ 300.00$

Health Insurance $\$ 35.00$

Misc. Total $\$ 335.00$

Figure 2: Working Budget for faculty travel for research and teaching, created by author. 


\section{The World at Home}

Taubman College of Architecture + Urban Planning

International Institute Exhibition, March 2018

\begin{tabular}{|c|c|c|c|}
\hline Item & Quantity & Price / unit & Cost \\
\hline \multicolumn{4}{|l|}{ Objects/Artifacts } \\
\hline Coasters & 50 & $\$ 1.30$ & $\$ 65.00$ \\
\hline Pens & 250 & $\$ 1.00$ & $\$ 250.00$ \\
\hline Mouse Pads & 25 & $\$ 5.00$ & $\$ 125.00$ \\
\hline Tote Bags & 50 & $\$ 10.00$ & $\$ 500.00$ \\
\hline Mugs & 8 & $\$ 15.00$ & $\$ 120.00$ \\
\hline Post-it Notes & 5 & $\$ 7.00$ & $\$ 35.00$ \\
\hline Puzzle & 2 & $\$ 17.00$ & $\$ 34.00$ \\
\hline \multicolumn{4}{|l|}{ Printing/Mounting } \\
\hline Book printing (City Analysis/Maps, Urban Design) & 4 & $\$ 100.00$ & $\$ 400.00$ \\
\hline Photo printing \& Mounting $\left(10^{\prime \prime} \times 10^{\prime \prime}\right.$, right wall) & 16 & $\$ 36.00$ & $\$ 576.00$ \\
\hline Photo printing \& Mounting $\left(10^{\prime \prime} \times 10^{\prime \prime}\right.$, left wall) & 14 & $\$ 36.00$ & $\$ 504.00$ \\
\hline Photo printing \& Mounting $\left(20^{\prime \prime} \times 20^{\prime \prime}\right.$, right wall) & 7 & $\$ 100$ & $\$ 700.00$ \\
\hline \multicolumn{4}{|l|}{ Graphics } \\
\hline Text Printed and Mounted on Wall (not window) & 1 & $\$ 200.00$ & $\$ 200.00$ \\
\hline Information Pamphlet (photo identification) & 50 & $\$ 5.00$ & $\$ 250.00$ \\
\hline \multicolumn{4}{|l|}{ Installation } \\
\hline Installation Assistants (2 student employees) & 20 & $\$ 15.00$ & $\$ 300.00$ \\
\hline Paint and patch wall (de-installation) & & & $\$ 100.00$ \\
\hline \multicolumn{4}{|l|}{ Extended Exhibition (into hallway) } \\
\hline Photo printing \& Mounting & 10 & $\$ 40.00$ & $\$ 400.00$ \\
\hline Hanging hardware & 1 & $\$ 30.00$ & $\$ 30.00$ \\
\hline Subtotal & & & $\$ 4,589.00$ \\
\hline Contingency + Shipping (5\%) & & & $\$ 227.95$ \\
\hline TOTAL & & & $\$ 4,816.95$ \\
\hline
\end{tabular}

Figure 3: Budget for the exhibition "The World-At Home," created by author. 


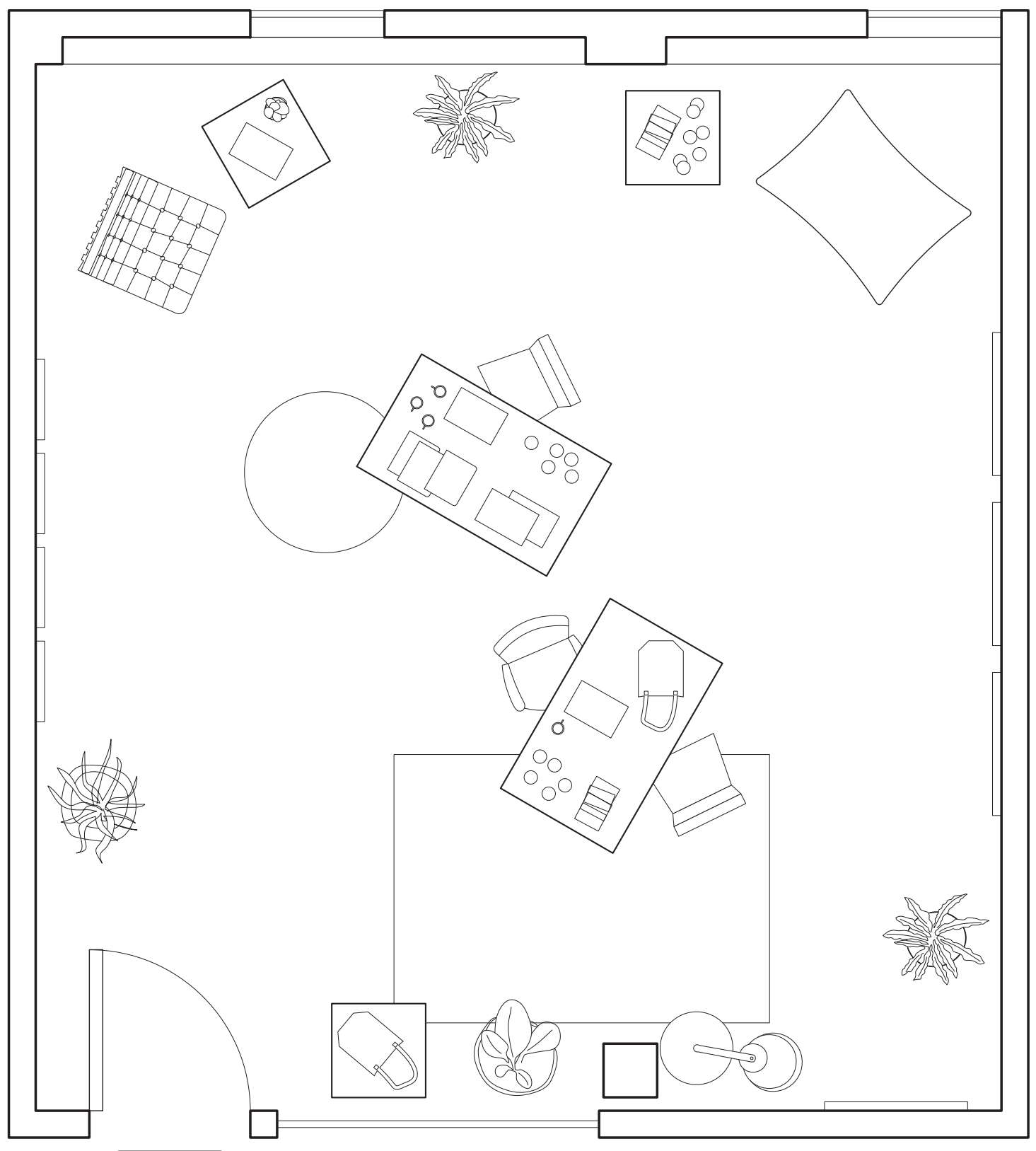

Figure 4: Plan for the exhibition "The World-At Home," created by author. 
Storefront Installation Budget

Materials

Item

Notes

Cost

Foam

EPS, High-Denisty 3\# Foam

$\$ 7,000$

Rubber

Recycled Rubber roll, \$3.84/sqft

$\$ 16,000$

Glue

Gorilla Glue, $\$ 50 / 1 \mathrm{lb}$ bottle

$\$ 1,000$

Cutting Tools

Xacto Knifes, Blades

$\$ 500$

Misc. Supplies

Super glue, scrapper

$\$ 700$

Material Total $\$ \mathbf{\$ 2 5 , 2 0 0}$

Lighting

\begin{tabular}{lll} 
Neon Light Fixtures & 6 Fixtures @\$300 each & $\$ 1,800$ \\
Custom Neon Storefront Sign & 1 Sign @ \$1,000 & $\$ 1,000$ \\
& \multicolumn{1}{c}{ Lighting Total } & $\mathbf{\$ 2 , 8 0 0}$
\end{tabular}

Labor

Design Assistant(s)

Fabrication Assistant(s)

Transportation/Shipping

\begin{tabular}{|c|c|}
\hline 9 weeks @\$14/hr & $\$ 5,000$ \\
\hline 9 weeks@\$14/hr & $\$ 5,000$ \\
\hline Truck rental, shipping costs & $\$ 2,000$ \\
\hline Labor Total & $\$ 12,000$ \\
\hline
\end{tabular}

Total $\$ 40,000$

Figure 5: Budget for a temporary storefront installation proposed for Detroit, Michigan, created by author. 